\title{
Modelling Anisotropic Diffusion and Elasto-Plastic Deformation during Lithiation of Silicon Nanowires
}

\author{
Ajaykrishna Ramasubramanian ${ }^{1}$, Vitaliy Yurkiv $^{1}$, Ali Najafi ${ }^{2}$, Ali Khounsay $^{3}$, Reza Shahbazian-Yassar $^{1}$, \\ Farzad Mashayek ${ }^{1}$ \\ ${ }^{1}$ Department of Mechanical and Industrial Engineering, University of Illinois at Chicago \\ Chicago, Illinois 60607, USA \\ mashayek@uic.edu \\ ${ }^{2}$ Ansys, Inc., Energy Centre of Excellence \\ Houston, Texas 77094, USA \\ ${ }^{3}$ Department of Physics, Illinois Institute of Technology \\ Chicago, Illinois 60616, USA
}

\section{Extended Abstract}

Crystalline silicon $(\mathrm{Si})$ is a promising anode material for a new generation of high capacity lithium (Li)-ion batteries due to its high energy density [1]. However, its utility is hampered by its large volumetric change (about 300\%) associated with the lithiation process. Recent experiments have demonstrated that this lithiation-induced volumetric expansion is highly anisotropic, with predominant expansion in the $\langle 110\rangle$ direction, but negligibly small along the $\langle 111\rangle$ direction [2]. This large anisotropic swelling and the induced stress cause mechanical failure and pulverization of the electrode resulting in a capacity fading. Thus, a detailed understanding of the stress evolution and the anisotropic kinetics of diffusion in such electrodes is crucial for the potential development of durable high capacity Li-ion batteries.

In this work, a generic framework to describe Li intercalation kinetics and the corresponding anisotropic structural changes in Si nanoelectrodes is presented. The governing equations are solved using finite element formulation using two different approaches. The first approach is based on a coupled thermal-structural model that treats temperature analogues to the diffusion process. The second approach is a phase-field model that accounts for the large deformation through a set of coupled phases-field and mechanics equations that are solved using the finite element method. Both models are generally applicable to any electrode system undergoing phase change and large elasto-plastic deformation. The developed constitutive models in the present work are first validated using the published data on isotropic lithiation in Si nanowire [3] and then the model has been extended to the orthotropic diffusion case.

The analysis of the lithium concentration evolution shows that the apparent anisotropic expansion could be attributed to the interfacial processes that accommodate large volumetric strains at the lithiation reaction front due to the crystallographic orientation. The structural changes in lithiated cylindrical Si nanowires are indicated by the formation of dumbbell-shaped cross section. By analysing the observed material evolution and stresses behind the reaction front, the importance of the structural relaxation, due to the plastic flow behind the moving phase boundary can be understood. On comparing the stresses behind the reaction front, it is observed that the stress induced during the lithiation process is dominant in the hoop direction and in the radial direction stress remains negligible. Despite the extensive plastic flow during the lithiation process, due to excessive deformation and anisotropic expansion, high stresses develop inside the lithiated region of the nanowire, leading to cracking or splitting of the single nanowire into sub-wires. The study sheds light on the lithiation-induced failure in nanowire-based electrodes. The modelling framework provides a basis for simulating the morphological evolution, stress generation, and material failure in high-capacity electrodes for the nextgeneration lithium-ion batteries.

\section{References}

[1] J. M. Tarascon and M. Armand, "Issues and challenges facing rechargeable lithium batteries," Nature, vol. 414, no. 6861, pp. 359-67, 2001.

[2] A. Magasinski, P. Dixon, B. Hertzberg, A. Kvit, J. Ayala, and G. Yushin, "High-performance lithium-ion battery 
anodes using a hierarchical bottom-up approach," Nat. Mater., vol. 9, p. 461, 2010.

[3] L. Chen, F. Fan, L. Hong, J. Chen, Y. Z. Ji, S. Zhang, T. Zhu, and L. Q. Chen, "A phase-field model coupled with large elasto-plastic deformation: application to lithiated silicon electrodes," J. Electrochem. Soc., vol. 161, no. 11, pp. F3164-F3172, 2014. 